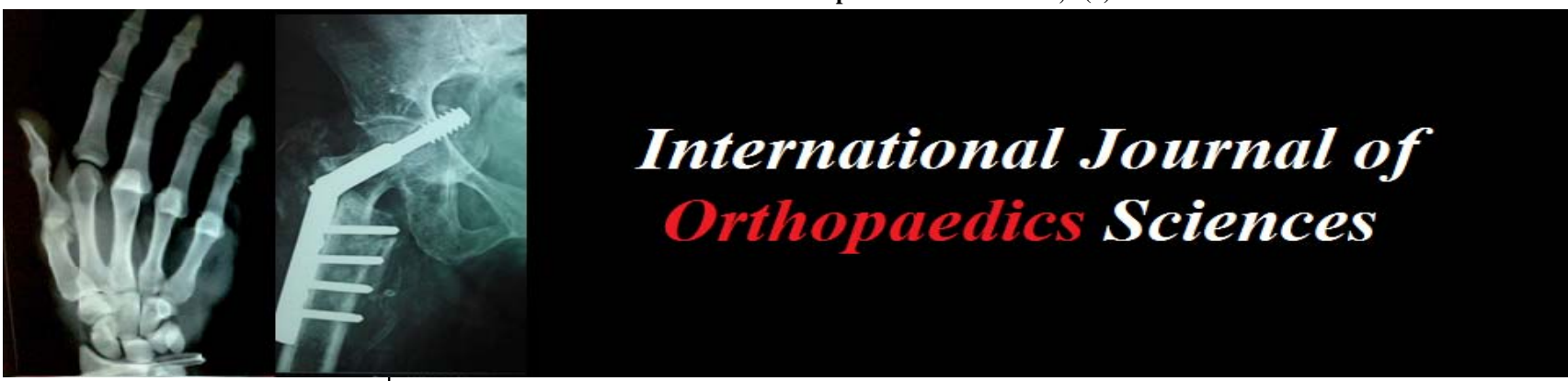

ISSN: $2395-1958$

IJOS c2017; 3(2): 901-906

(C) 2017 IJOS

www.orthopaper.com

Received: 10-02-2017

Accepted: 11-03-2017

Nabarun Saha

RMO Cum Clinical Tutor,

NRS Medical College, Kolkata,

Bengal, India

Kiran Kumar Mukhopadhyay Professor, NRS Medical College, Kolkata, Bengal, India

Anant Kumar Garg

Assisstant Professor,

NRS Medical College, Kolkata,

Bengal, India

Sanjay Kumar

Associate Professor,

NRS Medical College, Kolkata,

Bengal, India

Partha Sarathi Sarkar

Associate Professor,

NRS Medical College, Kolkata,

Bengal, India

Correspondence

Efstathios Drampalos

RMO Cum Clinical Tutor,

NRS Medical College, Kolkata,

Bengal, India

\section{Comparative study of debridement, fusion and instrumentation via single stage anterior or single stage posterior approach in dorsolumbar spinal tuberculosis}

\author{
Nabarun Saha, Kiran Kumar Mukhopadhyay, Anant Kumar Garg, \\ Sanjay Kumar and Partha Sarathi Sarkar
}

DOI: $\underline{\text { http://dx.doi.org/10.22271/ortho.2017.v3.i2j.100 }}$

\section{Abstract}

Background: There is controversy regarding approach for surgical treatment of dorsolumbar spinal tuberculosis. The aim of this study is to make a comparative study on the basis of clinico-serological, radiological, neurological, pain improvement and kyphotic angle correction between anterior and posterior surgery in dorsolumbar spinal tuberculosis.

Materials and methods: The study was performed on a cohort comprising of 40 patients treated surgically between Feb'09 to Jan'13. All these patients were analyzed and divided into two groups on the basis of surgical approach. Group I comprised 18 patients who underwent anterior debridement, decompression and instrumentation by either transthoracic transpleural or thoracoabdominal diaphragm cutting or retroperitoneal approach depending upon location of lesions. Group II comprised 22 patients who were operated by posterior transpedicular decompression, debridement and instrumentation with or without anterior interbody cage through midline posterior approach. Various parameters were assessed like surgical time, intra-operative blood loss, improvement in ESR, neurological recovery, correction of kyphosis and loss of correction of kyphosis, VAS pain score, functional outcome by Prolo's scale and fusion according to Bridwell criteria.

Results: Mean surgical time was more in Group I than Group II. Average blood loss was less in Group I. Clinico-serological outcome was better in Group I when compared to Group II. Regarding neurological outcome all patients improved at least 1 grade except one patient in Group II who deteriorated from Frankel D to Frankel C. Mean kyphotic angle correction was better in Group II with less loss of correction (LOC). Post-operative pain improvement, functional outcome and fusion based on Bridwell criteria were better in Group II compared to Group I.

Conclusion: Single stage posterior approach with transpedicular decompression, debridement, fusion and instrumentation is better than anterior approach owing to its better functional outcome, less morbidity, early mobilization and rehabilitation, better kyphotic correction and better maintenance of correction.

Keywords: Spinal tuberculosis, anterior approach, Posterior approach, Kyphosis correction

\section{Introduction}

Spinal tuberculosis is the commonest form of sketetal tuberculosis and it constitutes about 50 percent of all cases of tuberculosis of bone and joints ${ }^{[1]}$. Any part of the spinal column may be affected but dorsolumbar spine is most commonly involved. The development of modern, specific anti-tubercular drugs has revolutionized the treatment of tuberculosis. Spinal tuberculosis being a medical disease, chemotherapy is the mainstay of treatment. However, the disease typically has an insidious onset and slow progression. Patients usually seek attention weeks to months after onset of original symptoms and even if they present early, clinician may miss the diagnosis. Plain radiograms are not sufficient in early diagnosis and MRI is not routinely advised. In such scenario the diagnosis of spinal tuberculosis is difficult and it commonly presents at an advanced stage with dreaded complications like spinal deformity and neurological deficit which usually requires surgery $[2,3]$. Decompression of neural tissue, debridement of lesion followed by correction of spinal deformity and stabilisation with implants under cover of anti-tubercular chemotherapy has been shown to relieve pain, improve neurological impairment and prevent progression of deformity ${ }^{[4]}$. 
This remains the principle of surgical intervention. The commonly practiced surgeries in dorsolumbar spinal tuberculosis are:-

1. Anterior decompression/debridement + fusion with anterior instrumentation -i.e. anterior alone surgery

2. Anterior decompression/debridement + fusion with posterior instrumentation - i.e. combined anterior and posterior surgery

3. Posterior transpedicular decompression/debridement with posterior instrumentation and fusion - i.e. posterior alone surgery ${ }^{[5]}$

The selection of anterior versus posterior approach for surgical treatment of thoracolumbar tuberculosis is a matter of debate. The aims of this study are to carry out debridement along with fusion and instrumentation as per need and feasibility in selected patients of dorsolumbar spinal tuberculosis either through single stage anterior or through single stage posterior approach; evaluate their outcome in terms of clinico-serological criteria of disease control, radiological sign of healing, neurological and functional improvement, pain relief, correction of kyphotic deformity and prevention of progression of kyphotic deformity and make a comparative study between them.

\section{Materials and methods}

It is a retrospective and prospective study without any control. The study was performed on a cohort comprising of 40 patients (16 males: 24 females, with mean age 39.5 years, range 6-74 years) treated surgically between Feb'09 to Jan' 13. Inclusion criteria of the patients were: neurological deficit not responding or worsening with anti-tubercular drugs for 46 weeks, rapidly progressive gross neurology at initial presentation, bony destruction leading to kyphotic deformity ( $>40$ degrees), spinal instability ( $>50 \%$ collapse or A-P/lat translation), severe pain not responding to anti tubercular drugs and refractory cold abscess not responding or worsening with anti tubercular drugs. All these patients were analyzed and divided into two groups on the basis of surgical approach. Group I comprised 18 patients with mean age 40.9 years, (range 22-72 years), who underwent anterior debridement/ decompression with anterior instrumentation and fusion by:- transthoracic transpleural approach for thoracic lesions (D4-D11); thoracoabdominal diaphragm cutting approach for thoracolumbar junction (D11-L2) and retroperitoneal approach for lower lumbar lesions. Group II comprised 22 patients with mean age 38.2 years, (range 6-74 years), who were operated by posterior transpedicular decompression/ debridement with posterior instrumentation and fusion through midline posterior approach.

The distribution of patients according to vertebral lesion level is shown in TABLE 1 .

Table 1: distribution of pathologic vertebrae

\begin{tabular}{|c|c|c|c|}
\hline Group & Dorsal & Dorsolumbar & Lumbar \\
\hline Anterior (group i) & 9 & 6 & 3 \\
\hline Posterior (group ii) & 3 & 7 & 12 \\
\hline Total & 12 & 13 & 15 \\
\hline
\end{tabular}

Routine blood investigations, plain radiographs, and magnetic resonance imaging (MRI) studies were conducted before surgery for all patients. All patients received four anti tubercular chemotherapy minimum of 3 weeks prior to surgery.

The operative technique for each group is as follows.

\section{Group I (Anterior approach)}

All 18 patients underwent single stage anterior radical debridement, decompression, fusion and instrumentation. They were operated under general anesthesia in right lateral decubitus position through aforementioned approach according to the level of lesion. The diseased vertebra was reached. Thorough debridement and decompression carried out. Pus came from diseased vertebrae. It was extended as far as the dura to facilitate decompression of the cord. The endplates were curetted to reach the bleeding bone. Materials sent for histopathological examination and culture sensitivity. Titanium staple washer, screws, connecting rods and mesh cage impregnated with autologous iliac crest bone graft was used for reconstruction. Dorsal spinal tuberculosis with neurological involvement operated by anterior approach is demonatrated in Figure1.
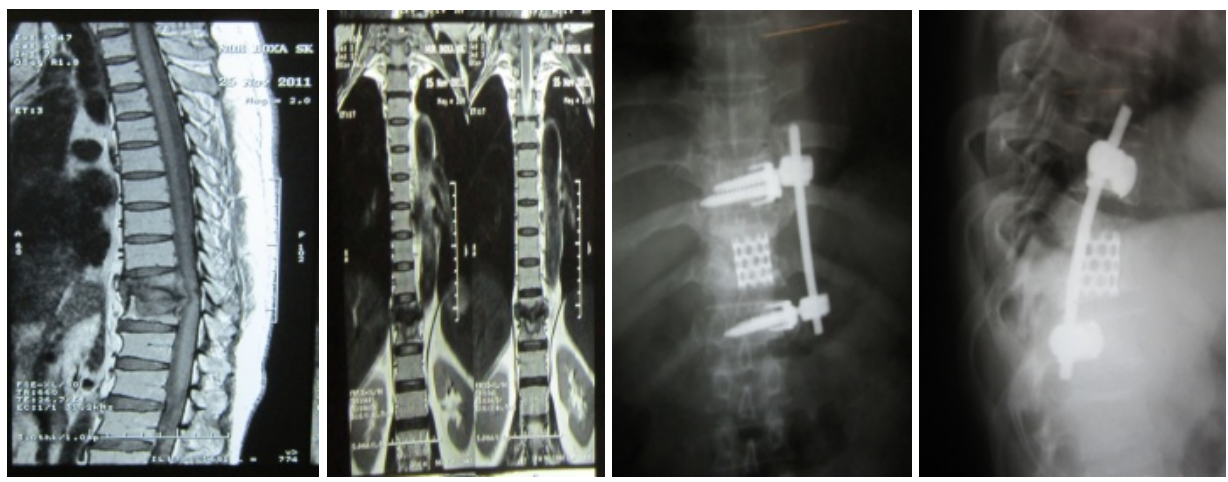

Fig 1: Preoperative MRI and post operative radiographs of D10-D11 caries spine with neurological involvement operated through anterior approach.

\section{Group II (Posterior approach)}

All 22 patients were operated in prone position under general anaesthesia through midline posterior approach. Pedicular screws were inserted into the proximal and distal stable vertebra under image intensifier guidance. Transpedicular decompression was carried out in the diseased vertebrae and materials sent for histopathological examination and culture sensitivity. Figure 2 demonstrates L2-L3 caries spine operated through posterior approach. 

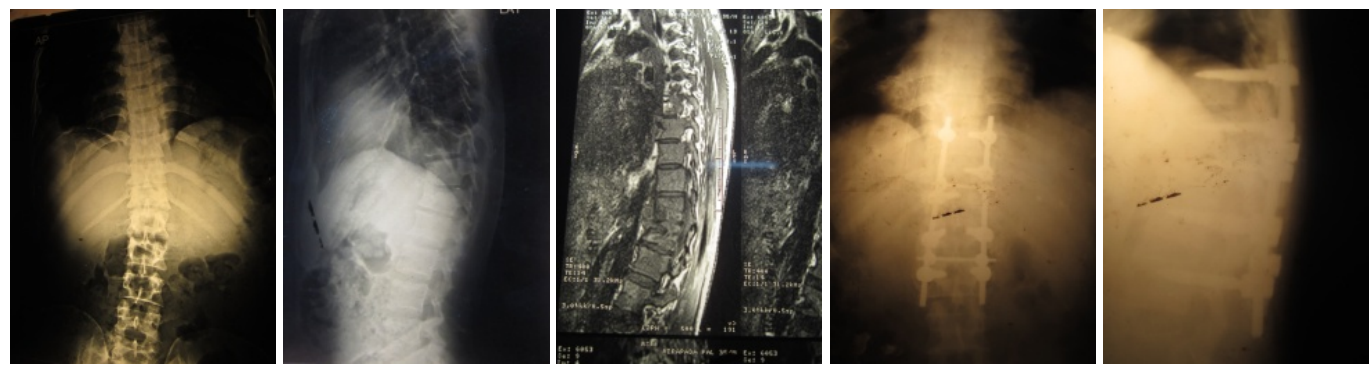

Fig 2: Preoperative X-ray and MRI and post operative radiographs of L2-L3 caries spine with neurological involvement operated through posterior approach.

In 2 cases of lumbar caries a mesh cage filled with autologous iliac crest bone graft is used from one side for defect reconstruction as demonstrated in Figure 3. Intratransverse bone grafting was done to facilitate fusion. Connecting rods \& cross link bars were applied.
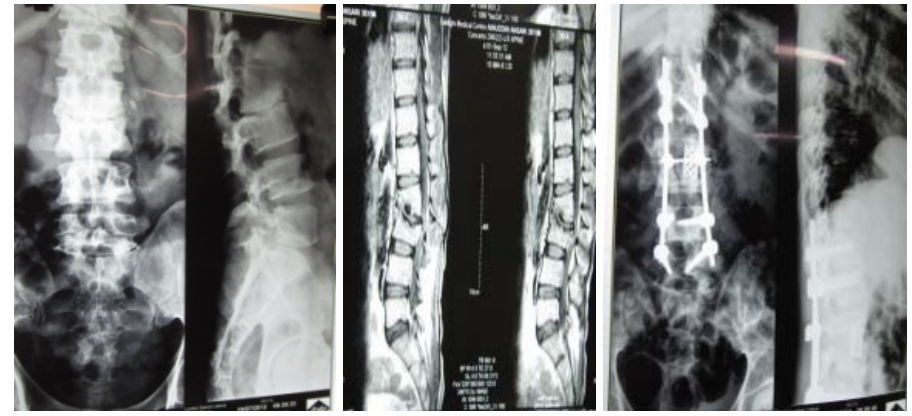

Fig 3: Preoperative X-ray and MRI and post operative radiographs of L2-L3 caries spine operated with anterior cage placement operated through posterior approach.

Broad-spectrum antibiotic was used intravenously for all patients 3 days postoperatively. All patients had drain removal on $2^{\text {nd }}$ day. Chest and limb physiotherapy and change of posture were started from $2^{\text {nd }}$ day. Chest drain in transthoracic approach of Group I were usually removed at $5^{\text {th }}$ day after proper chest $\mathrm{X}$ ray. Stitch removal was usually on the $14^{\text {th }}$ day. Proper bracing was used for 16-20 weeks postoperatively. When the neurology permitted, patients were out of bed from that day onwards. Anti tubercular drugs continued for 18 months. All cases were followed-up at 1, 3, 6, 9 and 12 months then annually. CT scans were performed at 12 months follow up for assessment of fusion. Mean follow up duration in the series was 28.6 months.

Various parameters were assessed between two groups like surgical time, intra-operative blood loss, post operative ICU care, improvement in ESR, neurological recovery, correction of kyphosis and loss of correction of kyphosis. Improvement of pain was assessed by VAS scale and functional outcome by Prolo's scale. Fusion assessment was done according to
Bridwell criteria.

\section{Results}

The most common site of involvement was lumbar spine $(37.5 \%)$ followed by dorsolumbar spine $(32.5 \%)$ and dorsal spine $(30 \%)$. Mean interval between the onset of symptoms and clinical presentation was 11.2 weeks (3-42 weeks). Neurological impairment is most common indication of surgery $(42.5 \%)$ followed by severe persistent pain not responding to conservative management (32.5\%). Both kyphosis $>40^{\circ}$ along with spinal instability and refractory cold abscess not responding to ATD shared $12.5 \%$ each. Mean surgical time was $3 \mathrm{hr} 05$ mins in Group I as compared to $2 \mathrm{hr}$ 35 mins in Group II. Average blood loss in Group I $427 \mathrm{ml}$ versus $552 \mathrm{ml}$ in Group II. Two patients in Group I required post operative ICU care as compared to none in Group II. Clinico-serological outcome was better in Group I when compared to Group II as depicted by improvement in ESR in TABLE 2.

Table 2: improvement in ESR

\begin{tabular}{|c|c|c|c|c|c|c|}
\hline \multicolumn{2}{|c|}{ ESR(mm/hr) } & $<40$ & $41-60$ & 61-80 & $81-100$ & $>100$ \\
\hline \multirow{3}{*}{$\begin{array}{l}\text { Anterior } \\
\text { (group I) }\end{array}$} & At Presentation & 4 & 2 & 5 & 5 & 2 \\
\hline & 2 wks Postop & 8 & 5 & 4 & 1 & 0 \\
\hline & At Final follow up & 13 & 4 & 1 & 0 & 0 \\
\hline \multicolumn{2}{|c|}{ ESR(MM/HR) } & $<40$ & $41-60$ & $61-80$ & $81-100$ & $>100$ \\
\hline \multirow{3}{*}{$\begin{array}{l}\text { Posterior } \\
\text { (group ii) }\end{array}$} & At Presentation & 9 & 5 & 5 & 3 & 0 \\
\hline & 2 weeks Postop & 11 & 7 & 4 & 0 & 0 \\
\hline & At Final follow up & 13 & 7 & 2 & 0 & 0 \\
\hline
\end{tabular}

Regarding neurological outcome all patients improved at least 1 grade as shown in TABLE 3 except one patient in Group II who deteriorated from Frankel D to Frankel C. 
Table 3: Improvement of neurological status in frankel grading

\begin{tabular}{|c|c|}
\hline \multicolumn{2}{|c|}{ Group I: (anterior approach) } \\
\hline At Presentation & At Final Follow Up \\
\hline \multirow{2}{*}{ Frankel B 2 } & $\mathrm{D}=1$ \\
$\mathrm{E}=1$ \\
\hline \multirow{2}{*}{ Frankel C 8 } & $\mathrm{D}=4$ \\
& $\mathrm{E}=4$ \\
\hline Frankel D 6 & $\mathrm{E}=6$ \\
\hline Frankel E 2 & $\mathrm{E}=2$ \\
\hline \multicolumn{2}{|c|}{ Group II (Posterior Approach) } \\
\hline At Presentation & At Final Follow Up \\
\hline \multirow{2}{*}{ Frankel C 4 } & $\mathrm{D}=1$ \\
& $\mathrm{E}=3$ \\
\hline \multirow{2}{*}{ Frankel D 8 } & $\mathrm{C}=1$ \\
& $\mathrm{D}=2$ \\
& $\mathrm{E}=5$ \\
\hline Frankel E 10 & $\mathrm{E}=10$ \\
\hline
\end{tabular}

Mean kyphotic angle correction was better in Group II (79.4\%) compared to Group I (69.5\%). Maximum kyphosis correction was achieved in dorsolumbar spine via posterior approach $\left(38^{\circ}\right)$. The percentage of immediate kyphotic correction at dorsolumbar spine was $91.7 \%$ in Group II versus $79.6 \%$ in Group I. Mean loss of correction (LOC) of initial kyphosis correction in Group I was $4.1^{\circ}$ and in Group II was $2.5^{\circ}$. Two weeks post-operatively pain improvement in mean VAS score was better in Group II (2.9) compared to Group I (4.2). Functional outcome was better in Group II with GOOD outcome in $81.8 \%$ compared to $72.2 \%$ in Group I. Based on Bridwell criteria fusion was better in Group II. $72.7 \%$ patients in Group II had Grade I fusion compared to $66.7 \%$ in Group I.

\section{Discussion}

There are few articles in literature comparing outcomes between single stage anterior and single stage posterior approach in surgical management of dorsolumbar spinal tuberculosis. We feel the reason may be that anterior and posterior instrumentation, respectively, have their own surgical indications; therefore their outcomes cannot be compared because patients require different levels of treatment. Nevertheless, we did this research because we believe our results will help determine which surgery - single stage anterior or single stage posterior - is best indicated for each individual patient.

Anterior approach is considered the gold standard for debridement and decompression in caries spine, which was popularized by Hodgson ${ }^{[6]}$ in 1960 . As stated by Jain et al ${ }^{[7]}$ since the vertebral body lies anterior and is commonly affected in tuberculosis, decompression and stabilization has to be done by approaching the spine anteriorly. However, Xiaobing $\mathrm{Pu}$ et al ${ }^{[8]}$, S.Basu et al ${ }^{[5]}$ and others have suggested posterior approach is sufficient for lesion debridement and those patients in whom the entire surgery can be done posteriorly should be done with bilateral transpedicular/extracavitatory decompression/debridement and instrumentation and fusion. Efficacy of debridement is best correlated by clinico-serological outcome. In this series clinico-serological outcome was better in Group I when compared to Group II although it is not statistically significant. The reasons may be either due to probable inadequate debridement of diseased body through posterior approach or because 2 patients in Group II discontinued ATD after poor compliance and their ESR raised after an intial fall post operatively. However, on starting ATD both of them responded well and their ESR are in waning phase. Moreover, with the advent of highly efficacious antitubercular drugs, indications for surgery have become more selective- less for the biological control of disease, but more for prevention and correction of deformities/complications, and for functional improvement ${ }^{[1]}$. Under cover of anti tubercular chemotherapy both the approaches are equally effective in disease eradication.

Instrumentation is necessary in caries spine whether it is approached through anteriorly or posteriorly. In this series, titanium alloy instruments were used owing to their relatively friendly nature towards the host bone and low infection rate. Mycobacterium tuberculosis has low adherence properties towards stainless steel [9], but its behaviour towards titanium has not yet been studied in detail. In this series only two patients of Group II had superficial wound infections which were managed by regular dressing and change in parenteral antibiotics. There was no incidence of deep infection. For anterior instrumentation, the use of a cage and bone grafts instead of a structural bone graft alone allows more rigid fixation construct and minimizes the risk of graft subsidence or dislodgement, that are well documented complications when structural bone graft alone is used ${ }^{[10]}$. Regarding posterior approach it was long been considered that removal of healthy posterior parts of vertebrae renders the vertebral column unstable and liable to pathological dislocation in the usual tuberculous lesion of the spine ${ }^{[1]}$. Recently, improved technology in the field of imaging has added a new dimension to the understanding of the surgical pathology of spinal tuberculosis [1, 11-16]. In views of Mehta \& Bhojraj (2001) simultaneous involvement of the anterior and posterior columns is more common than has been previously considered ${ }^{[17]}$. Involvement of the pedicle and facets are important factors in the genesis of instability, particularly if combined with destruction of a vertebral body at the same level. Thus, better understanding of biomechanics of spine, new concept of both column involvements in spinal tuberculosis and with the advancement in instrumentation system, sacrificing the intact posterior elements is no longer considered important for maintaining stability. Pedicle screws cross the pedicle, the strongest part of the vertebral body, providing three dimensional correction and stabilisation, which is much stronger than anterior instrumentation.

Kyphosis correction was better achieved in Group II patients who had undergone posterior transpedicular debridement, fusion and posterior instrumentation. A statistically significant difference has been found in mean kyphotic angle correction between anterior $(69.5 \%)$ and posterior approach (79.4\%) with $\mathrm{p}$ value $<0.05$. This correction in individual approach is much greater than the reports of Sundararaj et al ${ }^{[18]}(54.9 \%)$ and Elmi et al (53.4\%), however both of them used combined anterior and posterior approach. In 2 of the patients with more than two vertebral involvement in dorsolumbar spine and kyphosis $>40^{\circ}$ pedicle substraction osteotomy (PSO) has been performed through posterior approach (figure 3). 


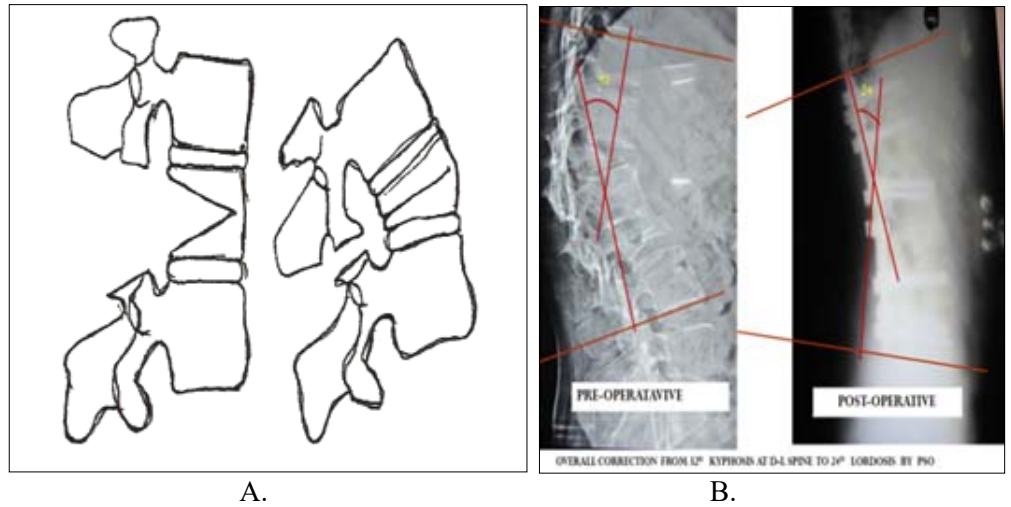

Fig 3: Kyphosis correction by Pedicle Substraction Osteotomy through posterior approach

This finding suggests that using a single stage posterior approach is as sufficient as a combined approach when correction of deformity is concerned similar to observations of Yuan Zheng Ma et al ${ }^{[19]}$ and Zhonghua et al ${ }^{[20]}$. The mean loss of correction (LOC) in this series is significantly less in Group II (posterior approach) when compared to Group I (anterior approach) with p value $<0.05$.

On observing neurological improvement all patients in our series improved by atleast 1 grade except one patient in Group II. The patient who deteriorated was of D12-L1 caries spine with neurological involvement frankel D \& kyphosis of $50^{\circ}$. Post operatively she deteriorated to frankel C. Patient was managed conservatively with Physiotherapy, brace \& Ankle Foot Orthosis. Neurology gradually improved to $3 / 5$ in $\mathrm{B} / \mathrm{L}$ EHL \& Ankle dorsiflexion. Other motor power became 5/5. However, on comparing the two groups complete neurological recovery was found in $82 \%$ in Group II when compared to $72 \%$ in Group I. Pain improvement and Functional outcome were better in posterior approach compared to anterior approach. Fusion at 1 year as assessed by Bridwell criteria based on CT scan imaging was better in posterior approach compared to Group I, similar to that of B. Garg et al ${ }^{[21]}$.

\section{Conclusion}

Thus for a given indvidual of dorsolumbar spinal tuberculosis single stage posterior approach with transpedicular decompression, debridement, fusion and instrumentation under the cover of anti tubercular chemotherapy may be considered favourable compared to anterior approach owing to its better functional outcome, less morbidity, early mobilization and rehabilitation, better kyphotic and saggital plane correction and better maintenance of correction. However, in dorsal spinal tuberculosis without kyphotic deformity, with single disc affection and large paravertebral abscess, anterior surgery is preferred as handling of the spinal cord at this level may be hazardous.

\section{References}

1. Tuli SM. Tuberculosis of the skeletal system (Bones, Joints, Spine and Bursal Sheaths) $4^{\text {th }}$ Edition, Jaypee Brothers.

2. Cormican L, Hammal R, Messenger J, Milbum HJ. Current difficulties in the diagnosis and management of spinal tuberculosis. Postgrad. Med. J. 2006; 82:46-51.

3. Turgut M. Spinal tuberculosis: Its clinical presentation, surgical management and outcome. A survey study on 694 patients. Neurosurg. Rev. 2001; 24:8-13.

4. Rajasekaran S, Shanmugasundaram TK. Prediction of the angle of gibbus deformity in tuberculosis of the spine. $\mathrm{J}$ Bone Joint Surg Am. 1987; 69:503-9.

5. Saumyajit Basu, Sandip Chatterjee, Bhattacharya MK. Efficacy and safety of instrumentation in Caries Spine. Indian Journal of Orthopedics. 2006; 40:78-81.

6. Hodgson AR, Stock FS. Anterior fusion for the treatment of tuberculosis of the spine: the operative findings and result of treatment in the first one hundred cases. J Bone and Joint Surg. 1960; 42-A:295-310.

7. Jain AK. Treatment of spine with neurological complications. Clin Orthop, 2002; 398:75-84.

8. Xiaobing Pu, Oiang Zhou, Oinyi He, Fei Dai, Jianzhong $\mathrm{Xu}$, Zehua Zhang et al. A posterior versus anterior surgical approach in combination with debridement, interbody autograft and instrumention for thoracic and lumbar tuberculosis.

9. Oga M, Arizone T, Takasita M, Sugioka Y. Evaluation of the risk of instrumentation as a foreign body in spinal tuberculosis: clinical and biologic study. Spine, 1993; 18:1890-1894.

10. Rajasekaran S, Shanmugasundaram TK. Prediction of the angle of gibbus deformity in tuberculosis of the spine. J Bone Joint Surg Am. 1987; 69:503-9.

11. Nussbaum ES, Rockswold GL, Bergman TA, Erickson DL, Seljeskog EL. Spinal tuberculosis: A diagnostic and management challenge. J Neurosurg. 1995; 83:243-7.

12. Benli IT, Akalin S, Kis M, Citak M, Kurtulus B, Duman E. The results of anterior fusion and instrumentation in idiopathic scoliosis. Eur Spine J 2000 ; 9(6) : 5005-515

13. Lifeso RM, Weaver P, Harder EH: Tuberculous Spondylitis in adults. J Bone joint Surg Am. 1985; 67:1405-13.

14. Chen W-J, Chen C-H, Shih C-H. Surgical treatment of tuberculous spondylitis: 50 patients followed for 2-8 years. Acta Orthop Scand. 1995; 66:137-42.

15. Boachie-Adjei O, Squillante RG. Tuberculosis of the spine. Orthop Clin North Am. 1996; 27:95-103.

16. Jain R, Sawhney S, Berry M. Computed tomography of vertebral tuberculosis: patterns of bone destruction. Clin Radiol, 1993; 47:196-9.

17. Mehta JS, Bhojraj SY. Tuberculosis of the thoracic spine. A classification based on the selection of surgical strategies. J Bone Joint Surg [Br]. 2001; 83-B:859-63.

18. Sundararaj GD, Behera S, Ravi V, Venkatesh K, Cherian $\mathrm{VM}$, Lee V. Role of posterior stabilization in the management of tuberculosis of the dorsal and lumbar spine. J Bone Joint surg (Br). 2003; 85(1):100-106.

19. Yuan Zheng Ma, Xu Cui, Hong Wei Li, Xing Chen, Xiao Jun Cai, Yi Bing Bai. Outcomes of anterior and posterior 
instrumentation under different surgical procedures for treating thoracic and lumbar spinal tuberculosis in adults. Int Orthop. 2012; 36(2):299-305.

20. Zhonghua Yi Xue Za Zhi. One-stage surgical management for spinal tuberculosis PubMed. 2003; 83(2):110-3. Article in Chinese. PMID 12812677

21. Bhavuk Garg, Pankaj Kandwal, Bidre Nagaraja Upendra, Ankur Goswami, Arvind Jayaswal. Anterior versus posterior procedure for surgical treatment of thoracolumbar tuberculosis: A retrospective analysis: IJO, Year, 2012; 46(2):165-170. 